\title{
GHRELIN ACTIVATION BY INGESTION OF MEDIUM-CHAIN TRIGLYCERIDES IN HEALTHY ADULTS: A PILOT TRIAL
}

\author{
Y. Yoshimura ${ }^{1}$, S. Shimazu ${ }^{2}$, A. Shiraishi ${ }^{3}$, F. Nagano ${ }^{4}$, S. Tominaga ${ }^{5}$, T. Hamada ${ }^{4}$, M. Kudo ${ }^{2}$, \\ Y. Yamasaki ${ }^{4}$ S. Noda ${ }^{6}$, T. Bise ${ }^{4}$
}

\begin{abstract}
Objective: To investigate the efficacy of dietary supplementation of medium-chain triglycerides (MCTs) and its effects on ghrelin activation in healthy adults. Methods: The present study examined two protocols with six healthy volunteers: 1) 12-hour profiles of the plasma levels of acylated and desacyl ghrelin without MCT ingestion, and 2) changes in serum ghrelin levels after oral ingestion of $45 \mathrm{~g} /$ day of MCTs for 1 week. Results: At baseline, serum acylated and desacyl ghrelin levels were $18.2 \pm 10.3$ and $77.1 \pm 23.4 \mathrm{fmol} / \mathrm{mL}$, respectively. The ratio of acylated/desacyl ghrelin was $19 \%$. There were no significant differences in the 12-hour profiles of acylated and desacyl ghrelin. Significant increases were observed in all sampling times of serum acylated ghrelin after 1-week MCTs ingestion. The ratio of acylated/desacyl ghrelin increased to 37.7\%. Conclusions: Oral ingestion of MCTs increased serum acylated ghrelin levels in healthy adults, suggesting that MCTs administration stimulates food intake.
\end{abstract}

Key words: Ghrelin, acylation, medium-chain triglycerides (MCT), sarcopenia, cachexia.

\section{Introduction}

"Anorexia of aging" is defined as loss of appetite and reduced food intake in old age, and may be associated with decline in skeletal muscle mass, energy expenditure, and physical activity that occur in later years $(1,2)$. Decreased skeletal muscle mass is related to malnutrition, length of hospital stay, morbidity, and mortality (3, 4 ); malnutrition is frequent in populations with high morbidity and burden of care. Appetite loss is the major cause of malnutrition in older adults and sometimes difficult to control under established nutritional management settings, especially in clinical settings.

Ghrelin is a novel growth hormone (GH)-releasing peptide widely distributed throughout the gastric mucosa and is made up of 28 amino acids; it was first described in 1999 (5). Ghrelin exhibits orexigenic effects and complex metabolic activities through the GH-independent mechanism, leading to the augmentation of skeletal muscle mass and suppression of energy expenditure and

1. Department of Rehabilitation Medicine, Kumamoto Rehabilitation Hospital, Kumamoto, Japan; 2. Department of Nutritional Management, Kumamoto Rehabilitation Hospital, Kumamoto, Japan; 3. Department of Dental Office, Kumamoto Rehabilitation Hospital, Kumamoto, Japan; 4. Department of Rehabilitation, Kumamoto Rehabilitation Hospital, Kumamoto, Japan; 5. Department of Clinical Laboratory, Kumamoto Rehabilitation Hospital, Kumamoto, Japan; 6. Department of Nursing, Kumamoto Rehabilitation Hospital, Kumamoto, Japan

Corresponding Author: Yoshihiro Yoshimura, Department of Rehabilitation Medicine, Kumamoto Rehabilitation Hospital, 760 Magate, Kikuyo-Town, Kikuchi-County, Kumamoto, 869-1106, Japan; E-mail: hanley.belfus@gmail.com; Tel: +81-96-232-3111; Fax: +81-96-232-3119 inflammation $(6,7)$. Therefore, there is growing interest regarding the identification of ghrelin as a potentially valid and well-tolerated anabolic and anti-catabolic treatment for sarcopenia, cachexia, and other wasting disorders.

Ghrelin mainly exist in two forms: active (acylated ghrelin) and inactive (desacyl ghrelin). The third amino acid residue of ghrelin, serine, is modified by octanoic acids, C8:0 medium-chain triglycerides (MCTs); acylation is essential for the biological activity of ghrelin. While desacyl ghrelin is suggested to have limited effects on GH-receptor under physiological conditions $(8,9)$, acylated ghrelin corresponds to approximately $20 \%$ of the total circulating ghrelin, and is responsible for the biological effects of ghrelin (10), indicating that acylation is a vital step for the biological activity of ghrelin.

Therefore, it is hypothesized that ghrelin is acylated via ingestion of MCTs, and this could be a promising treatment option for malnutrition in older adults; however, evidence regarding its efficacy is very limited. In the current study, we investigate the efficacy of dietary supplementation of MCTs and its effects on ghrelin activation in healthy adults.

\section{Materials and Methods}

\section{Participants}

The current study was approved by the Institutional Research Ethics Committee of Kumamoto Rehabilitation 
Hospital (Kumamoto, Japan), and was performed in accordance with the ethical standards established in the 1964 Declaration of Helsinki and later amendments. All participants provided written informed consent in advance. We recruited six healthy volunteers who were working at the hospital in 2017, comprising 3 women and 3 men with mean age of $38 \pm 8$ years and mean body mass index (BMI) of $22.0 \pm 1.3 \mathrm{~kg} / \mathrm{m} 2$. At enrollment, the following criteria were applied: 1) normal body weight with BMI of $20-25 \mathrm{~kg} / \mathrm{m} 2$. The following exclusion criteria were adopted: 1) presence of diseases requiring treatment physically or psychologically, including dyslipidemia and diabetes mellitus; 2) taking any supplements or drugs; and 3) presence of appetite alteration. The participants' body composition were analyzed using bioelectrical impedance analysis (InBody S10; InBody, Tokyo, Japan).

\section{Study protocol}

The present study examined two protocols. First, 12-hour profiles of the serum levels of acylated and desacyl ghrelin without MCT ingestion were investigated. Second, changes in serum ghrelin levels after oral ingestion of $45 \mathrm{~g} /$ day of MCTs for 1 week were measured. Therefore, the study period of the current trial was designed to be 2 weeks [Fig. 1]. At baseline, the participants were evaluated and advised strictly on their daily lifestyle, including food intake, physical activity, and sleeping time, all of which were reported to potentially alter plasma ghrelin levels $(11,12)$. Each participant received a standardized meal protocol to control energy intake, set as daily energy intake of 30-35 kcal/ $\mathrm{kg}$ (body weight), supervised by a registered dietitian throughout the study period. Physical activity level (working during daytime) and sleeping time (6-7 hours at night) were standardized and supervised by a medical doctor throughout the study period.

To examine whether ingestion of MCTs affects serum ghrelin activation, serum ghrelin levels were measured after participants orally ingested $45 \mathrm{~g} /$ day of MCTs for 1 week. Participants were instructed to take one tablespoon (15 ml) of MCT oil added to each meal (breakfast, lunch, and dinner) every day, which accounts for $45 \mathrm{~g}(45 \mathrm{ml})$ of MCT ingestion a day. The MCTs used in this study were 100\% pure MCT oil (Nissin MCT Oil HC, The Nisshin OilliO Group, Ltd., Tokyo, Japan), and per $10 \mathrm{ml}$ of the oil consisted of $8.9 \mathrm{kcal}$ energy, $0 \mathrm{~g}$ protein, $10 \mathrm{~g}$ lipid, $0 \mathrm{~g}$ carbohydrate, and $10 \mathrm{~g} \mathrm{MCT.}$

\section{Blood sampling and assay}

Blood samples for the measurement of serum ghrelin were taken from the antecubital vein after 30 minutes of resting, and were drawn at 07:00, 10:00, 14:00, 17:00, and 19:00 hours to identify 12-hour profiles of serum ghrelin levels. Blood sampling was performed at baseline and after 1 week of MCT ingestion to measure the changes in serum ghrelin levels, complete blood counts and laboratory data. Serum acylated and desacyl ghrelin levels were measured by enzyme-linked immunosorbent assay (SRL, Inc., Tokyo, Japan).

\section{Statistical analysis}

All analyses were performed using SPSS version 21 for Windows. Results are presented as mean \pm standard deviation (SD). Comparisons were performed using a paired t-test before and after 1 week of daily oral ingestion of MCTs and one-way repeated measures ANOVA for changes in ghrelin 12-hour profiles, after which a post-hoc analysis was performed for before and after comparisons of the effects of MCT ingestion. $\mathrm{P}$ values of $<0.05$ were considered statistically significant.

\section{Figure 1}

Study protocol. The present study examined two protocols. First, 24-hour profiles of the plasma levels of acylated and desacyl ghrelin without MCT ingestion were investigated. Second, changes in plasma ghrelin levels after oral ingestion of $45 \mathrm{~g}$ / day of MCTs for 1 week were measured. The study period of the current trial was designed to be 2 weeks. MCT: medium-chain triglyceride

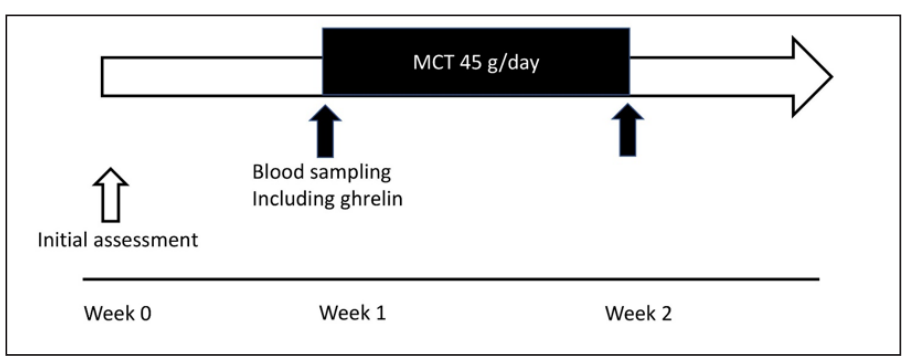

\section{Results}

\section{Clinical characteristics}

Table 1 presented the clinical characteristics of all the participants, showing that the study participants were neither lean nor obese, and that they were a homogenous group of healthy adults.

\section{Baseline plasma ghrelin level}

At baseline, in the morning, serum acylated and desacyl ghrelin levels were $18.2 \pm 10.3 \mathrm{fmol} / \mathrm{mL}$ and $77.1 \pm 23.4 \mathrm{fmol} / \mathrm{mL}$, respectively. The ratio of acylated ghrelin to desacyl ghrelin was $19 \%$. There were no significant differences in the 12-hour profiles of acylated and desacyl ghrelin, although acylated ghrelin tended to increase before meals and decreased after meals, without statistical significance [Fig. 2]. 
Table 1

Clinical characteristics of study participants and changes in parameters before and after 1-week MCT ingestion

\begin{tabular}{|c|c|c|c|c|}
\hline & & Baseline & Post MCT intake & $\mathrm{p}$ \\
\hline Age & year & $38 \pm 8$ & - & - \\
\hline Sex, male & $\mathrm{n}$ & 3 & - & - \\
\hline female & $\mathrm{n}$ & 3 & & \\
\hline Height & $\mathrm{cm}$ & $164.2 \pm 3.4$ & & \\
\hline Weight & $\mathrm{kg}$ & $59.3 \pm 4.1$ & - & - \\
\hline Body mass index & $\mathrm{kg} / \mathrm{m}^{2}$ & $22.0 \pm 1.3$ & - & - \\
\hline Lean body mass & $\mathrm{kg}$ & $46.6 \pm 5.1$ & - & - \\
\hline Skeletal muscle mass & $\mathrm{kg}$ & $19.4 \pm 2.2$ & - & - \\
\hline Skeletal muscle mass index & $\mathrm{kg} / \mathrm{m} 2$ & $7.1 \pm 0.6$ & - & - \\
\hline Fat mass & $\mathrm{kg}$ & $13.1 \pm 2.1$ & - & - \\
\hline Fat mass & $\%$ & $21.5 \pm 4.1$ & - & - \\
\hline Acylated ghrelin* & $\mathrm{fmol} / \mathrm{ml}$ & $18.2 \pm 10.3$ & $38.3 \pm 23.8$ & 0.016 \\
\hline Desacyl ghrelin* & $\mathrm{fmol} / \mathrm{ml}$ & $77.7 \pm 23.4$ & $63.3 \pm 21.1$ & 0.089 \\
\hline WBC & count & $7033 \pm 900$ & $6900 \pm 1000$ & 0.563 \\
\hline Hemoglobin & $\mathrm{g} / \mathrm{dl}$ & $14.4 \pm 0.8$ & $14.4 \pm 0.9$ & 0.918 \\
\hline Total protein & $\mathrm{g} / \mathrm{dl}$ & $7.5 \pm 0.1$ & $7.5 \pm 0.1$ & 0.341 \\
\hline Albumin & $\mathrm{g} / \mathrm{dl}$ & $4.2 \pm 0.1$ & $4.2 \pm 0.1$ & 0.175 \\
\hline Total cholesterol & $\mathrm{mg} / \mathrm{dl}$ & $199 \pm 25.1$ & $200 \pm 26$ & 0.883 \\
\hline Triglyceride & $\mathrm{mg} / \mathrm{dl}$ & $101 \pm 9.1$ & $103 \pm 8.9$ & 0.412 \\
\hline Fasting glucose & $\mathrm{mg} / \mathrm{dl}$ & $93.5 \pm 5.5$ & $94.5 \pm 6.0$ & 0.690 \\
\hline CRP & $\mathrm{mg} / \mathrm{dl}$ & $0.07 \pm 0.07$ & $0.04 \pm 0.02$ & 0.264 \\
\hline
\end{tabular}
o'clock.

\section{Effect of MCT ingestion on plasma ghrelin and serum data}

Significant increases were observed in all sampling times of the 12-hour profiles of serum acylated ghrelin after 1-week oral administration of $45 \mathrm{~g} /$ day of MCTs [Fig. 2], while significant decreases were observed in 4 out of 5 sampling times for serum desacyl ghrelin. The ratio of acylated ghrelin to desacyl ghrelin increased to $37.7 \%$ in the morning after completion of 1 week of MCT ingestion. The increase in serum acylated ghrelin peaked early in the morning but not after meals. We observed that 1 week of MCT administration did not alter total cholesterol, triglyceride, or fasting glucose levels.

\section{Adverse effects}

There were no adverse effects reported throughout the study, including digestive symptoms, serum lipid profiles, and fasting glucose levels.

\section{Discussion}

Here we report the results of our study examining the effect of oral MCT ingestion on the acylation of serum ghrelin levels in healthy adults. We demonstrate that ghrelin was acylated after MCT ingestion and that the ratio of acylated/desacyl ghrelin level increased. This finding suggests that MCT consumption may trigger acylation (activation) of ghrelin, leading to increased appetite, positive energy balance, and improved nutritional status in malnourished older adults or those at the risk of malnutrition.

Some studies demonstrated that MCT was a good substrate for the conversion of ghrelin to active ghrelin under normal physiological conditions, without affecting total ghrelin concentrations (13-16); however, the samples examined in those studies were animals (e.g., mice, rats, lactating dairy cows, and chickens). For example, Lemarié et al. conducted a study using rats and reported no significant increase in acylated ghrelin levels, despite MCT administration; however, a significant increase was observed in the acylated / total ghrelin ratio (16). 
Figure 2

12-hour profiles and changes of acylated ghrelin and desacyl ghrelin before and after 1-week ingestion of MCTs. MCT: medium-chain triglyceride. ${ }^{*}, \mathrm{p}<0.05$; ${ }^{* *}, \mathrm{p}<0.001$ for ghrelin level before vs. after MCTs administration

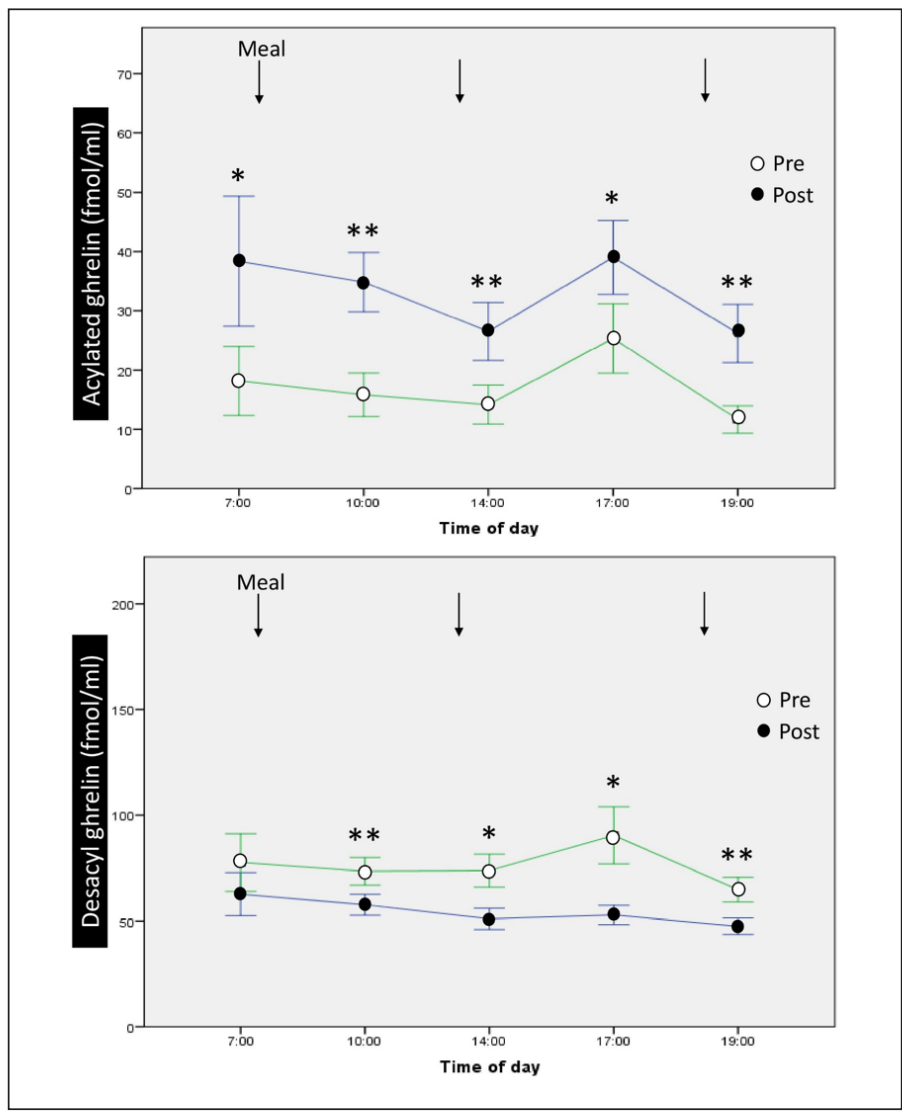

Human studies regarding this subject are limited. There are only two studies available in the literature targeting patients with disease-related nutritional disorders, on the associations of MCT administration and serum acylated ghrelin levels. Ashitani et al. (17) reported that MCTs supplemented via a combination of oral and nasogastric tube administration increased serum acylated ghrelin levels among inpatients diagnosed with anorexia nervosa (AN). In addition, they observed a dosedependent relationship between MCT administration and serum acylated ghrelin levels in AN patients. Kawai et al. (18) reported the positive effects of orally ingested MCTs on serum acylated ghrelin levels in patients with chronic respiratory diseases. We believe that the above patient groups may have decreased appetites due to such diseases, with increased reactivity of serum ghrelin levels due to MCT administration.

Exogenous ghrelin administration is an effective appetite stimulant, confirmed by several studies published since 2000. Infusion and bolus of acylated ghrelin have consistently been shown to increase appetite and / or food intake in healthy adults $(19,20)$, obese adults (21), patients with chronic pulmonary diseases (22), cancer $(23,24)$, heart failure (25), gastric dysfunction (26), and chronic renal failure receiving maintenance dialysis (27). Although there is strong evidence regarding the effects of exogenous ghrelin administration on appetite, circulating GH, adrenocorticotropic hormone, cortisol, glucose, and prolactin in diverse patient cohorts, there is limited evidence demonstrating the positive effects of ghrelin on body composition, pulmonary function, cardiac function, and bone metabolism. Furthermore, some adverse effects due to ghrelin administration were reported in $20 \%$ of the study participants, including flushing, gastric rumbles, thirst, somnolence, vertigo, fatigue, and change in mood $(28,29)$. Therefore, activation of ghrelin via dietary supplementation of MCTs instead of exogenous ghrelin administration may be an effective and safe treatment option for diverse populations with appetite alteration.

To the best of our knowledge, this is the first study to demonstrate that ghrelin is activated via orally ingested MCTs in healthy adults. This finding suggests that acylation (activation) of ghrelin via oral ingestion of MCTs stimulates food intake; induces positive energy balance; and aids in the treatment of older patients with malnutrition, cachexia, and sarcopenia, where little evidence is currently available (30).

Our study had some limitations. First, this study included small samples, 3 women and 3 men, without discussing the sex difference. This might limit the generalizability of the results. Second, another control group, such as the long-chain triglycerides (LCTs) administered group, was lacking. Thus, the single effect of MCTs could not be clarified in the current study design. Therefore, further large-scale and high-quality studies are needed to address these limitations.

In conclusion, oral ingestion of MCTs increased serum acylated ghrelin levels in healthy adults. The present result suggests that treatment with MCTs may be a promising treatment option for patients with malnutrition, as well as sarcopenia and cachexia, while further large-scale and high-quality studies are needed.

Funding sources: This study was supported by Nisshin OilliO Group, Ltd., Japan. Nisshin OilliO Group, Ltd. had no control over the interpretation, writing, or publication of the research.

\section{Conflict of interest: No conflict of interest.}

Author contributions: YY: supervising, concept, data collecting, interpretation, manuscript drafting. SS, AS: concept, interpretation, manuscript drafting. FN, ST, $\mathrm{TH}, \mathrm{MK}, \mathrm{YY}, \mathrm{SN}, \mathrm{TB}$ : concept, interpretation, data collecting, manuscript drafting.

Ethical standards: We conducted the study in accordance with the Declaration of Helsinki, and the study was approved by the ethics committee of Kumamoto Rehabilitation Hospital. We obtained written informed consent from all participants.

\section{References}

1. Wilson MMG, Morley JE. Invited Review: Aging and energy balance. J Appl Physiol 2003;95:1728-1736.

2. O'Shea E, Trawley S, Manning E, et al. Malnutrition in Hospitalised Older Adults: A Multicentre Observational Study of Prevalence, Associations and Outcomes. J Nutr Health Aging 2017;21:830-836. 
3. Cruz-Jentoft AJ, Baeyens JP, Bauer JM, et al. Sarcopenia: European consensus on definition and diagnosis: Report of the European Working Group on Sarcopenia in Older People. Age Ageing 2010;39:412-423

4. Yoshimura Y, Wakabayashi H, Bise T, Tanoue M. Prevalence of sarcopenia and its association with activities of daily living and dysphagia in convalescent rehabilitation ward inpatients. Clin Nutr. 2017; doi: 10.1016/j. clnu.2017.09.009. [Epub ahead of print]

5. Kojima M, Hosoda H, Date Y, et al. Ghrelin is a growth-hormone-releasing acylated peptide from stomach. Nature 1999;402:656-660.

6. Barazzoni R, Bosutti A, Stebel M, et al. Ghrelin regulates mitochondrial-lipid metabolism gene expression and tissue fat distribution in liver and skeletal muscle. Am J Physiol Endocrinol Metab 2005;288:E228-E235.

7. Anker SD, Coats AJ, Morley JE. Evidence for partial pharmaceutical reversal of the cancer anorexia-cachexia syndrome: the case of anamorelin. J Cachexia Sarcopenia Muscle 2015;6:275-277.

8. Stengel A., Tache Y. Ghrelin - a pleiotropic hormone secreted from endocrine x/a-like cells of the stomach. Frontiers in Neuroscience 2012;6:24.

9. Mirzaie Bavil F., Mohaddes G., Ebrahimi H., et al. Ghrelin increases lymphocytes in chronic normobaric hypoxia. Advanced Pharmaceutical Bulletin 2014;4:339-343.

10. Castaneda T. R., Tong J., Datta R., et al. Ghrelin in the regulation of body weight and metabolism. Frontiers in Neuroendocrinology 2010;31:44-60.

11. Garin MC, Burns CM, Kaul S, Cappola AR. Clinical review: The human experience with ghrelin administration. J Clin Endocrinol Metab 2013;98:18261837.

12. Pradhan G, Samson SL, Sun Y. Ghrelin: much more than a hunger hormone. Curr Opin Clin Nutr Metab Care 20113;16:619-624.

13. Nishi $Y$, Hiejima $H$, Hosoda $H$, et al. Ingested medium-chain fatty acids are directly utilized for the acyl modification of ghrelin. Endocrinology 2005;146:2255-2264

14. Fukumori R, Sugino $\mathrm{T}$, Shingu $\mathrm{H}$, et al. Ingestion of medium chain fatty acids by lactating dairy cows increases concentrations of plasma ghrelin. Domest Anim Endocrinol 2013;45:216-223

15. Yamato M, Sakata I, Wada R, et al. Exogenous administration of octanoic acid accelerates octanoylated ghrelin production in the proventriculus of neonatal chicks. Biochem Biophys Res Commun 2005;333:583-589.

16. Lemarié F, Beauchamp E, Dayot S, et al. Dietary Caprylic Acid (C8:0) Does Not Increase Plasma Acylated Ghrelin but Decreases Plasma Unacylated Ghrelin in the Rat. PLoS One 2015;10:e0133600.

17. Ashitani J, Matsumoto N, Nakazato M. Effect of octanoic acid-rich formula on plasma ghrelin levels in cachectic patients with chronic respiratory disease.
Nutr J 2009;8:25. doi: 10.1186/1475-2891-8-25.

18. Kawai K, Nakashima M, Kojima M, et al. Ghrelin activation and neuropeptide $\mathrm{Y}$ elevation in response to medium chain triglyceride administration in anorexia nervosa patients. Clin Nutr ESPEN 2017;17:100104.

19. Druce MR, Neary NM, Small CJ, et al. Subcutaneous administration of ghrelin stimulates energy intake in healthy lean human volunteers. Int J Obes (Lond) 2006;30(2):293-296.

20. Schmid DA, Held K, Ising M, et al. Ghrelin stimulates appetite, imagination of food, GH, ACTH, and cortisol, but does not affect leptin in normal controls. Neuropsychopharmacology 2005;30:1187-1192.

21. Huda MS, Dovey T, Wong SP, et al. Ghrelin restores 'lean-type' hunger and energy expenditure profiles in morbidly obese subjects but has no effect on postgastrectomy subjects. Int J Obes (Lond) 2009;33:317-325.

22. Kodama T, Ashitani J, Matsumoto N, et al. Ghrelin treatment suppresse neutrophil-dominant inflammation in airways of patients with chronic respiratory infection. Pulm Pharmacol Ther 2008;21:774-779.

23. Yamamoto K, Takiguchi S, Miyata H, et al. Randomized phase II study of clinical effects of ghrelin after esophagectomy with gastric tube reconstruction. Surgery 2010;148:31-38.

24. Lundholm K, Gunnebo L, Körner U, et al. Effects by daily long term provision of ghrelin to unselected weight-losing cancer patients: a randomized double-blind study. Cancer 2010;116:2044-2052.

25. Nagaya N, Moriya I, Yasumura Y, et al. Effects of ghrelin administration on left ventricular function, exercise capacity, and muscle wasting in patients with chronic heart failure. Circulation 2004;110:3674-2679.

26. Akamizu T, Iwakura H, Ariyasu H, et al. Clinical Study Team. Repeated administration of ghrelin to patients with functional dyspepsia: its effects on food intake and appetite. Eur J Endocrinol 2008;158:491-498.

27. Ashby DR, Ford HE, Wynne KJ, et al. Sustained appetite improvement in malnourished dialysis patients by daily ghrelin treatment. Kidney Int 2009;76:199-206.

28. Pradhan G, Samson SL, Sun Y. Ghrelin: much more than a hunger hormone. Curr Opin Clin Nutr Metab Care 2013;16:619-624.

29. Garin MC, Burns CM, Kaul S, Cappola AR. Clinical review: The human experience with ghrelin administration. J Clin Endocrinol Metab 2013;98:18261837.

30. Yoshimura $\mathrm{Y}$, Wakabayashi H, Yamada M, et al. Interventions for Treating Sarcopenia: A Systematic Review and Meta-Analysis of Randomized Controlled Studies. J Am Med Dir Assoc 2017;18:553.e1-553.e16. 